\title{
An Extended SISa Model for Sentiment Contagion
}

\author{
Zhifeng Liu, ${ }^{1}$ Tingting Zhang, ${ }^{1}$ and Qiujun Lan $^{2}$ \\ ${ }^{1}$ College of Management and Economics, Tianjin University, Tianjin 300072, China \\ ${ }^{2}$ Business School, Hunan University, Changsha 410082, China
}

Correspondence should be addressed to Qiujun Lan; lanqiujun@hnu.edu.cn

Received 17 April 2014; Accepted 2 July 2014; Published 16 July 2014

Academic Editor: Chuangxia Huang

Copyright (C) 2014 Zhifeng Liu et al. This is an open access article distributed under the Creative Commons Attribution License, which permits unrestricted use, distribution, and reproduction in any medium, provided the original work is properly cited.

\begin{abstract}
One of the main differences between sentiment and infectious diseases is that the former one has two opposite infectious states: positive (optimistic) and negative (pessimistic), while the latter one has not. In this paper, based on the SISa model, we consider this issue and propose a new model of sentiment contagion called the SOSa-SPSa model. The results of both numerical and agent-based simulations show that our model could explain the process of sentiment contagion better than that of Hill et al. (2010). Further analysis shows that both the numbers of optimistic and pessimistic individuals will increase with the probability of spontaneity or contagion and decrease with the probability of recovery. Potential applications of this model in financial market have also been discussed.
\end{abstract}

\section{Introduction}

Social emotion (or sentiment) is the result of the interaction between individuals, but how does this interaction take place and ultimately form the social emotion? Maybe the study of emotional contagion can give an answer for this question. Emotional contagion can be simply defined as a situation where every individual's emotion could be affected by others' emotion, and the whole process is usually spontaneous, unconscious, and uncontrolled [1]. The previous studies on emotional contagion are mainly concentrated in the field of psychology and sociology [2-5]; however, in recent years, this issue has been also discussed in financial market.

In the field of psychology and sociology, the behavior of emotional contagion has been studied in many papers $[6,7]$. The most representative works are SIS and SIR models [811], which belong to the classic epidemic models and are designed to study the infection of diseases. But now these models have been used to solve the problem of emotion contagion. Dodds and Watts (2005) discuss these two models in a unified framework [12]. Hill et al. (2010), who have considered the spontaneous generation process of emotion, explore the issues of emotional contagion on the basis of SISa model and use the data from the Framingham Heart
Study to estimate the model parameters [13]. As we all know, social networks are very complex [14-17], and the research of the complex networks is also the new trend of research on emotion contagion [18-20]. For example, Zhao et al. (2014) establish an emotion contagion model in complex networks and find that the tendency of emotion variation in the BA scale-free network is almost the same as that in the homogeneous network [21]. Another research method called computational model has been in use recently $[5,22,23]$. For example, Fu et al. (2014) simulate the dynamic process of emotion contagion based on the SIR model and find that infection frequency will increase with the average crowd density [24].

Investor sentiment is a special kind of individual emotion, which originates in people's psychological behavior, such as risk preference $[25,26]$. Sentiment contagion in the financial field is also a promising research topic (the sentimental process is similar to the emotional process, both of which have some common characteristics, but when we use the word "sentiment" in financial market, it contains more meanings related to asset prices. However, we are indeed not to distinguish the difference between these two words in this paper, and in the next part, we use the word "sentiment" instead of "emotion"). In recent years, scholars' 
understanding of investor sentiment contagion has already been developed to a certain scope on the macro level [2729]. Baker et al.'s (2012) research about contagion of investor sentiment is a representative work [30]. Chang et al. (2012) have also studied the problem of global sentiment contagion and the influencing factors of it, and they find the capital flow is a channel of contagion, but not the only one [31]. Overall, the research on sentiment contagion still needs further study, especially on the contagion mechanism and channel.

Using classical epidemic models to mathematically describe the sentimental contagion process can help us to understand and predict generation and spread of sentiment. But this method at least has a defect at present; that is, when treating sentiment as infectious disease, we need to mention that sentiment in real life has two opposite contagious states: positive (optimistic) and negative (pessimistic), but the disease only has one infectious state, which is the "infected" status in SIS or SIR model. For this reason, the usual epidemic model is not very suitable to be used to describe the process of sentiment contagion directly. Being different from the existing researches, this paper extends the SISa model with considering the two states of sentiment and builds a new model called SOSa-SPSa model. The main reason why we choose the SISa model is that, comparing with the classic SIS model, the SISa model involves an automatic process of sentiment generation, which is consistent with our intuition that sentiment can arise spontaneously. In our new model, both of the two opposite sentiment states are similar to the infectious state in SISa model. By treating optimism and pessimism as contagious, we divide the process of sentiment contagion into two processes: susceptible-optimistic-susceptible (SOS) and the susceptible-pessimistic-susceptible (SPS), and each of these processes is similar to the susceptible-infectedsusceptible (SIS) process.

The rest of this paper is divided into 3 sections. After providing some background information about sentimental contagion and giving a literature review in Section 1, we introduce our model in Section 2. Section 3 analyzes the equilibrium solutions of the new model by using both numerical and agent-based simulations. Finally, Section 4 summarizes the main conclusions of this paper and outlines some potential applications in further studies.

\section{The SOSa-SPSa Model}

According to whether the infector can become immune to the virus, the most classic epidemic models can be divided into two categories: one is SIS model; the other is SIR model. In the classic SIS model, an individual only switches between the two states: the susceptible $(S)$ and infected $(I)$. If considering the immune status which exists after some diseases' recovery, then the model can be transformed to SIR model. In this paper, we will regard sentiment as one kind of diseases, which can be transmitted among people. As we all know, individual sentiment is innate, spontaneous, and susceptible, and people face difficulty in being immune to others' sentiment. Therefore, we discuss the problem of sentimental contagion based on the SISa model, which is an extend model of SIS model with an automatic sentiment generation process.

The SISa model is proposed by Hill et al. (2010), and it considers the spontaneous generation process of sentiment [13]. The basic framework of the model is as in Figure 4, where $S$ and $I$ represent the susceptible people and the infected people, respectively, $q$ is the probability of the process of the susceptible transform into the infected, including both the spontaneous and infectious processes, and $g$ is the probability that the infected can recover back to the susceptible.

The characteristics of sentiment are very complex, and simply treating sentiment as a single status is not good enough for us to understand sentiment well. So, based on the SISa model above, this paper divides the infected status into two states: the optimistic $(O)$ and the pessimistic $(P)$.

And both the classic SIS model and SIR model have two assumptions (Hill et al., 2010): first, the more the susceptible are exposed to the infected, the more the probability the susceptible become to the infected; second, the probability of the infected people's recovery is irrelevant to the number of infected individuals. Hill et al. (2010) further consider the probability that the susceptible spontaneously convert to the infected, which means that no matter whether contacted with the infected or not, the susceptible will automatically transform into the infected at a certain probability, and it is consistent with the spontaneously generated pattern of sentiment. When considering dividing the sentiment into optimism and pessimism, the two assumptions discussed above are still applicable but not complete. Therefore, we add some other assumptions as follows.

(1) There are three kinds of sentiment states: the optimistic, the pessimistic, and the susceptible.

(2) The more the number of the optimistic whom the susceptible contacted with, the greater the probability that it will become optimistic; the more the number of the pessimistic whom the susceptible contacted with, the greater the probability that it will become pessimistic.

(3) The probability that the optimistic or the pessimistic recover back to the susceptible is irrelevant to the number of the optimistic or the pessimistic.

(4) The susceptible will spontaneously transform into the optimistic or the pessimistic at a certain probability.

In addition, because of the lack of understanding the transmission mechanism between optimism and pessimism, and for simplified analysis too, this paper assumes that the optimistic and the pessimistic can only transform into the susceptible but cannot transform into each other directly.

Based on the assumptions above, this paper analyzes the sentimental contagion process by considering the opposite sentiment states, and similar to Hill et al. (2010) [13], the processes can be mainly described in Figure 5 .

In Figure 5(a), the model depicts the process of the transformation between the optimistic and the susceptible. (1) The susceptible will not change to the optimistic due to their connections with the pessimistic. (2) When the susceptible 
one contacts with the optimistic ones, there is a probability of $\beta_{\mathrm{O}}$ for him or her to become an optimistic one. (3) Regardless of whom the susceptible one contacts with, there is a probability of $\alpha_{O}$ for him or her to become optimistic spontaneously. (4) Regardless of whom the optimistic one contacts with, there is a probability of $g_{O}$ for him or her to revert to the susceptible status.

Similarly, as we can see in Figure 5(b), the model describes the transformation process between the pessimistic and the susceptible. (1) The susceptible will not become pessimistic due to their connections with the optimistic. (2) When the susceptible one contacts with the pessimistic ones, there is a probability of $\beta_{P}$ for him or her to become a pessimistic one. (3) Regardless of whom the susceptible one contacts with, there is a probability of $\alpha_{P}$ for him or her to become pessimistic automatically. (4) Regardless of whom the pessimistic one contacts with, there is a probability of $g_{P}$ for him or her to recover to the susceptible status.

In brief, the individual mainly experiences two conversion processes: susceptible-optimistic-susceptible (SOS) and susceptible-pessimistic-susceptible (SPS), both of which include an automatic process of sentiment generation. Therefore, this paper names the new model as SOSa-SPSa model.

Figure 6 is a simple description for the basic framework of the SOSa-SPSa model, and different from the SISa model, the parameters $q_{\mathrm{O}}$ and $q_{P}$, which express the probabilities that the susceptible transform into the optimistic and the pessimistic, respectively, have new features. When considering the optimistic or pessimistic status of sentiment, the susceptible can contact with the optimistic and the pessimistic simultaneously, but in this situation, which status the susceptible will transform into? And how large the probability is? In order to discuss these problems conveniently, this paper assumes that the total number of individuals is $N$, and among them, the number of the optimistic is $O$, the number of the pessimistic is $P$, and the number of the susceptible is $S$, which satisfy the equation $O+P+S=N$. We also simply assume that the processes of the susceptible transform into the optimistic and the pessimistic are independent; thus the SOSa-SPSa model can be written as follows:

$$
\begin{gathered}
\frac{d S}{d t}=g_{O} O+g_{P} P-\left(\alpha_{O}+\alpha_{P}\right) S-\left(\beta_{O} O+\beta_{P} P\right) S . \\
\frac{d O}{d t}=-g_{O} O+\alpha_{O} S+\beta_{O} O S \\
\frac{d P}{d t}=-g_{P} P+\alpha_{P} S+\beta_{P} P S \\
O+P+S=N .
\end{gathered}
$$

Let $d S / d t=0, d O / d t=0$, and $d P / d t=0$, and we can get the equilibrium points of these differential equations. If $\alpha_{O}+\beta_{O} O \neq 0$ and $\alpha_{P}+\beta_{P} P \neq 0$, it is easy to obtain that $S=g_{O} O /\left(\alpha_{O}+\beta_{O} O\right)$ and $S=g_{P} P /\left(\alpha_{P}+\beta_{P} P\right)$. Thus, in the equilibrium state, the quantity relation between the number of the optimistic and the number of the pessimistic is given by

$$
\frac{g_{\mathrm{O}} O}{\alpha_{O}+\beta_{\mathrm{O}} O}=\frac{g_{P} P}{\alpha_{P}+\beta_{P} P} .
$$

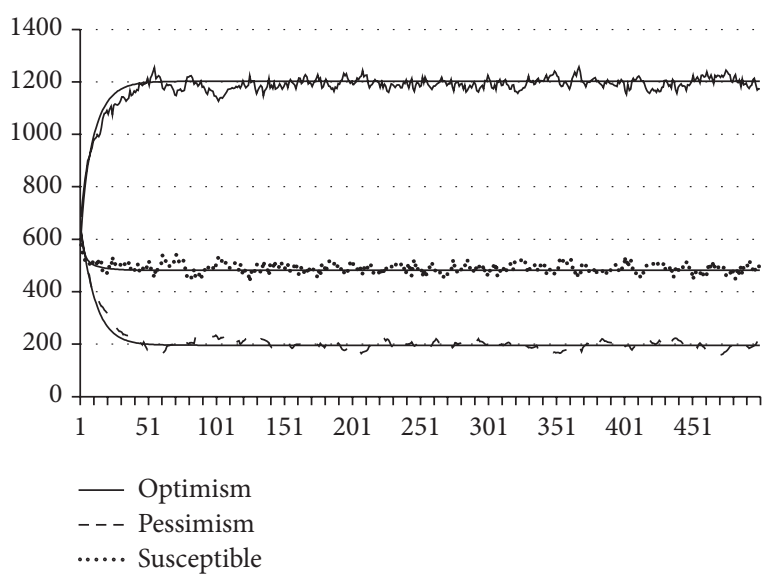

FIGURE 1: Equilibrium process of sentiment contagion.

\section{Model Simulations}

3.1. Equilibrium: Numerical and Agent-Based Simulations. The differential equation is popular in the relevant theory, but the solutions of these differential equations are usually complicated [32-34]; therefore we analyze the process of sentiment contagion by using the numerical and agentbased simulations. At first, we have to set the parameters, which usually depend on the specific situation. In this paper, according to the estimated results from Hill et al. (2010), we set the key parameters as follows: $\alpha_{O}=0.18, \beta_{O}=0.02$, $g_{\mathrm{O}}=0.088, \alpha_{P}=0.04, \beta_{P}=0.04$, and $g_{P}=0.13$. And we set the initial values of the number of three statuses as $O=626$, $P=626$, and $S=628$, so $N=1880$. In fact, the choice of initial values has no effect on the final results. Additionally, it is important to note that we do not assume the population as well mixed, but it has an average degree of 4 .

The final equilibrium states from numerical and agentbased simulations are shown in Figure 1, and we can find that the number of each state reaches a constant value by using numerical simulation $(O=1202.21(63.95 \%), P=$ $196.13(10.43 \%)$, and $S=481.66(25.62 \%)$ ), which is nearly identical to the results of the agent-based simulation. These results are almost consistent with the results from Hill et al. (2010), who have reported the proportion of each status. And these results show that our model is reasonable and can explain the process of sentiment contagion well.

\subsection{The Effect of Different Parameters on the Equilibrium.} There are six parameters in our model, which makes the equilibrium results very complex. Therefore, in order to facilitate the follow discussion, we assume that there is no difference between the optimistic and the pessimistic sentiment contagion processes. In fact, when the corresponding parameters are equal, the results of the two processes are symmetric. That is to say, when the equilibrium state has been reached, there will be the same number of the optimistic and the pessimistic. Therefore, at the beginning of the analysis in this section, we only discuss the related characteristics about the pessimistic. It means that this paper only discusses how 




(a)

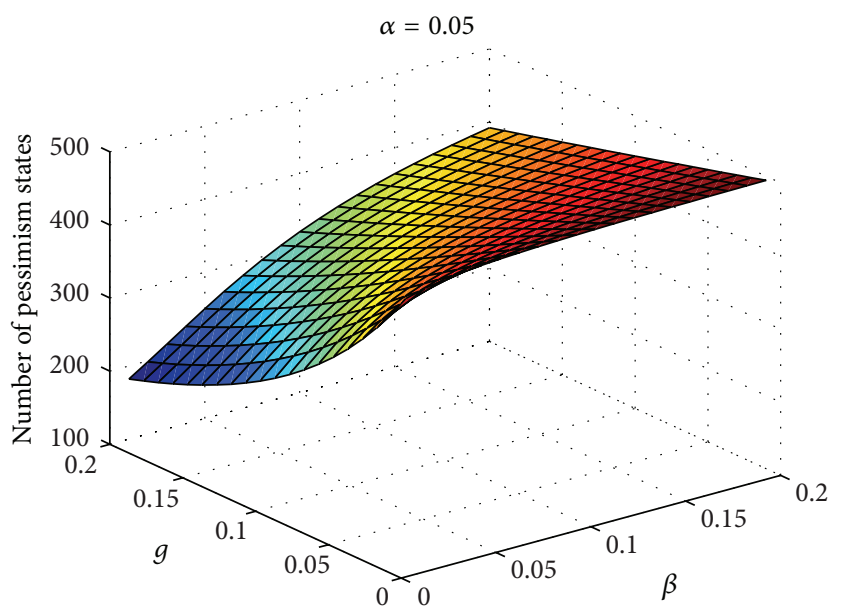

(c)

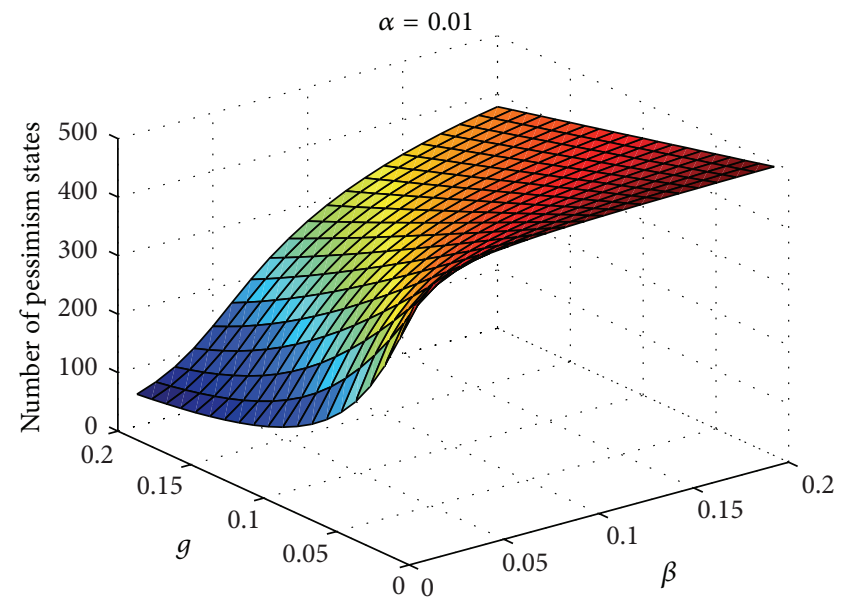

(b)



(d)

FIGURE 2: The influence of main parameters on the equilibrium.

the change of key parameters, which is associated with the pessimistic sentiment, affects the quantity of the pessimistic. In other words, we will discuss how the change of $\alpha_{P}, \beta_{P}$, and $g_{P}$ affects the equilibrium results. Meanwhile, in order to make the simulation results more intuitive, we set $N=$ 1000. In fact, we find that in the process of the simulation, the setting of other parameters has no effect on the basic conclusions of this paper.

Figure 2 shows how the pessimism's recovery speed and its transmission speed influence the equilibrium number of the pessimistic under different parameters. Simply speaking, the faster the speed of the spontaneous generation is, the higher the equilibrium number of the pessimistic is. And the number of the pessimistic in equilibrium is positively related to the transmission speed but negatively related to the recovery speed. This is the same with our intuition. The interesting thing is that when the speed of the spontaneous generation and the probability of the susceptible getting infected are both at a low level, the speed of recovery in different intervals may have obvious different effects on equilibrium quantity.

In practice, the contagion rate of pessimism is often bigger than that of optimism, but how does this difference influence the equilibrium? In order to further study this issue, we relax the assumptions above and take the unequal contagion rates into consideration. From Figure 3, we can find that when the speed of spontaneous generation is very small or even near 0 (it is also close to the SIS model which does not consider the spontaneous generation of sentiment), the tiny difference between the contagion speeds of pessimism and optimism may lead to a huge difference between the numbers of the final equilibrium quantity of each sentiment state. It means that when the contagion speed of pessimism is bigger than that of optimism, the former one will hold the dominant position quickly. But when the mechanism of sentiment's spontaneous generation exists, the above phenomenon would not be so obvious. This can be understood as follows: the mechanism of sentiment's spontaneous generation would do an important and stable job to avoid the unlimited spread 


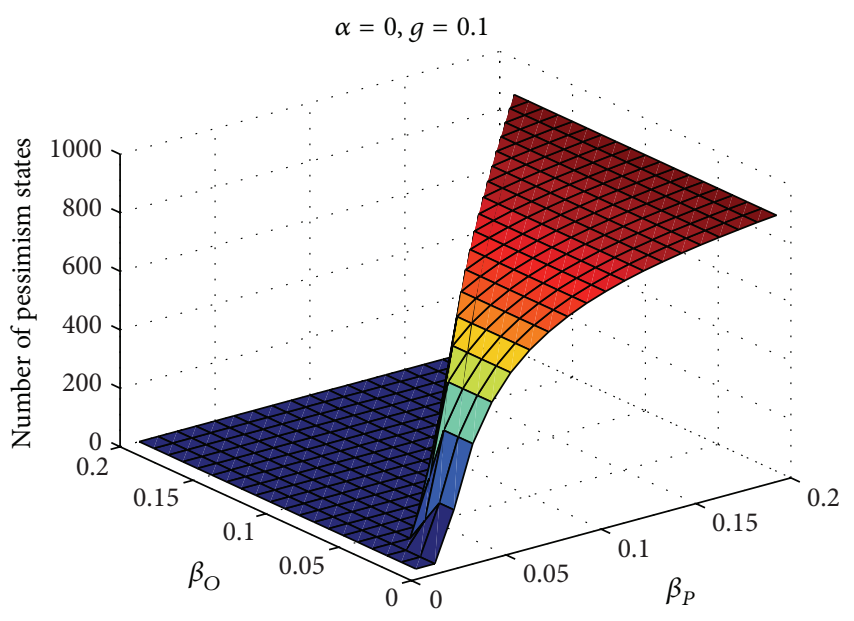

(a)

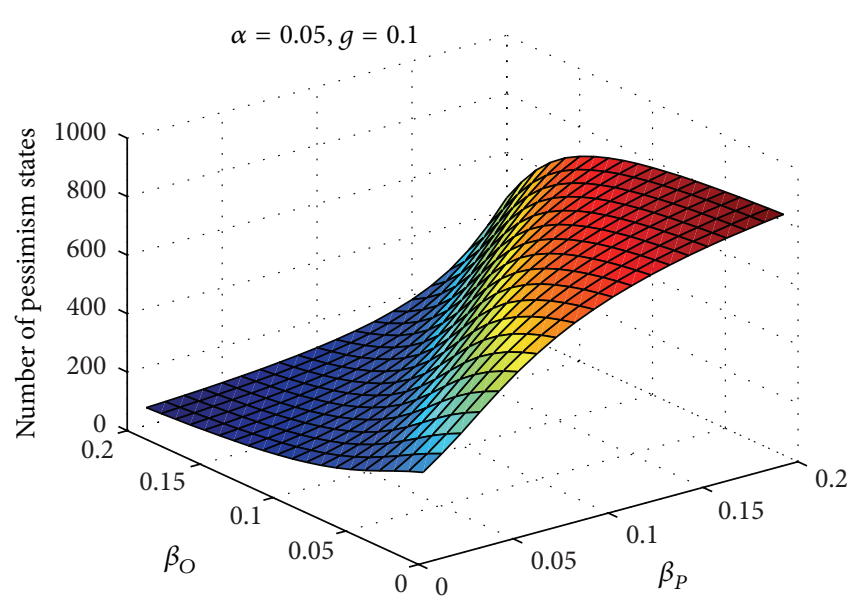

(c)



(b)



(d)

FIGURE 3: The influence of different infection probabilities on the equilibrium.

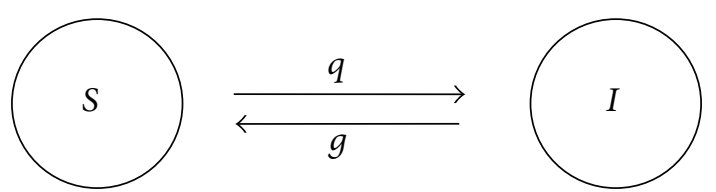

FigURE 4

of single sentiment, especially the negative sentiment. It also enlightens us about the importance of cultivating a good generation mechanism of optimistic sentiment in our society.

We also simulate our model by using agent-based model, and the limited experimental results show that the above numerical solutions are also stable.

\section{Discussions and Conclusions}

This paper extends the classic SISa model, proposes a new framework model considering both the optimistic and pessimistic states, and constructs a SOSa-SPSa model. The simulations of the new model show that the results can match with the experimental data of Hill et al. (2010) well. From the further analysis of the equilibrium results affected by the key parameters, we can discover that the equilibrium quantity of the pessimistic is only related to the pivotal parameters, while irrelevant to the initial values of each sentiment group.

The SOSa-SPSa model proposed by this paper can well portray the process of sentiment contagion involving both the optimistic and pessimistic states, and the results are also consistent with the limited experimental data. However, the lack of the stability analysis of the equilibrium of this model is the limitation of this paper. Although this paper already analyzes the equilibrium of the SOSa-SPSa model, the stability of the equilibrium has not been discussed mathematically yet. Actually, the SOSa-SPSa model has six key parameters, which makes the analysis of the stability of the equilibrium point become a very difficult job. And we just have some infinite tests to observe the stability of this model under the different parameters settings, which show that the above equilibrium points are stable. The simulation results of agent-based model also suggest that the equilibrium 


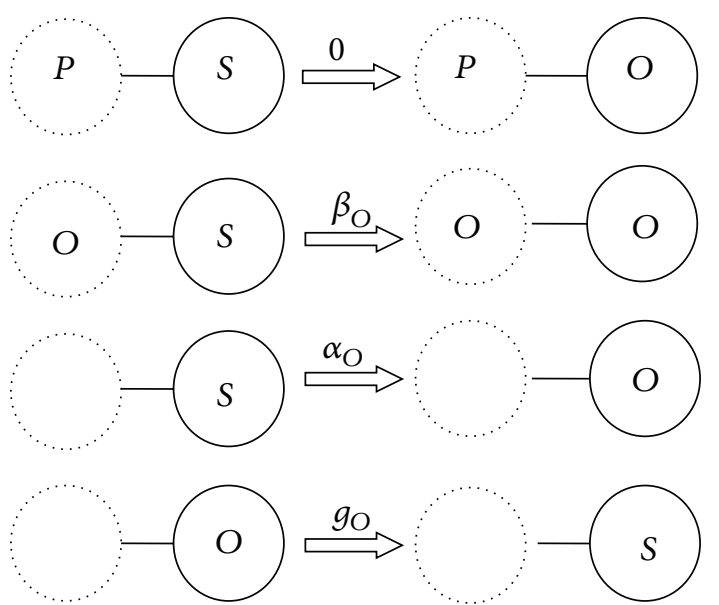

(a)

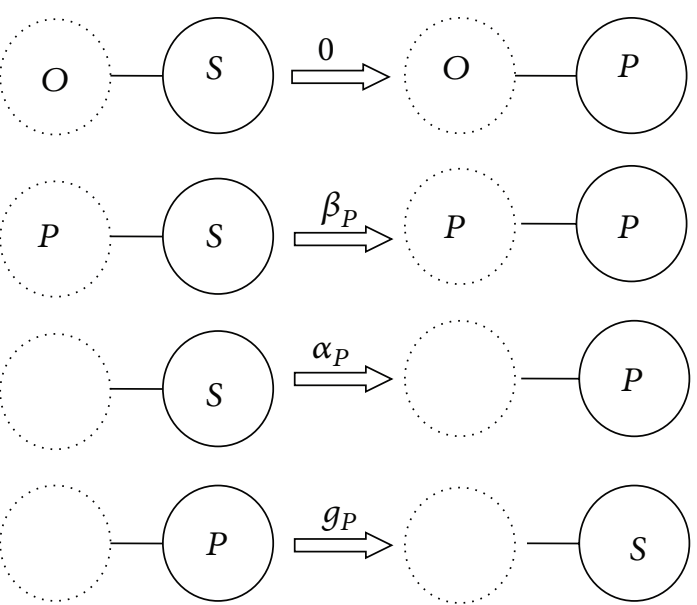

(b)

FIGURE 5

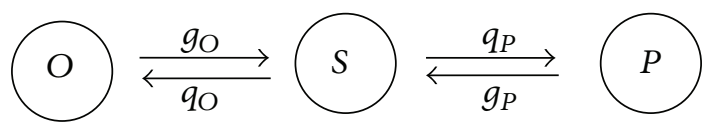

FIGURE 6

solution is stable, but it is still necessary to discuss the stability of the equilibrium model in a strict framework. We also have not considered the transformation mechanism between the optimistic and the pessimistic, which will be included in the next step of our work.

This SOSa-SPSa model can be used to study the investor sentiment contagion in the field of behavioral finance, but if you want to apply it to this topic, there are still some difficulties exist. We argue that the general sentiment contagion model in the field of psychology and sociology can only be used as a base model for investor sentiment contagion, but the deeper study of the characteristics of investor sentiment contagion is also very important and therefore necessary $[35,36]$. If you want to further consider the issue of the investor sentiment contagion based on our model, you will need to think deeply about the commonness and differences between the definition of "emotion" and "investor sentiment" in both fields of social psychology and behavioral finance. This is because the investor sentiment not only has some common features of social mood but also could be profoundly affected by many market factors, such as the special operation mechanism of financial markets [37], the diffusion pattern of market information, the characteristic of complex network among investors, and the people's risk attitude [38, 39], and the presence of these factors may lead the contagion process of investor sentiment to present different characteristics from those in the field of sociology and psychology. Therefore, in our future research, we will further study the contagion characteristics of investor sentiment based on the microscopic model, which helps us to understand the generation mechanism of the investor sentiment and the relationship between investor sentiment and asset price more profoundly.

\section{Conflict of Interests}

The authors declare that there is no conflict of interests regarding the publication of this paper.

\section{Acknowledgment}

This research is supported by the National Science Foundation of China (NSFC) (no. 71171076).

\section{References}

[1] K. R. Olson, "A literature review of social mood," The Journal of Behavioral Finance, vol. 7, no. 4, pp. 193-203, 2006.

[2] B. Parkinson and G. Simons, "Affecting others: Social appraisal and emotion contagion in everyday decision making," Personality and Social Psychology Bulletin, vol. 35, no. 8, pp. 1071-1084, 2009.

[3] S. D. Pugh, "Service with a smile: emotional contagion in the service encounter," Academy of Management Journal, vol. 44, no. 5, pp. 1018-1027, 2001.

[4] U. Hess and S. Blairy, "Facial mimicry and emotional contagion to dynamic emotional facial expressions and their influence on decoding accuracy," International Journal of Psychophysiology, vol. 40, no. 2, pp. 129-141, 2001.

[5] T. Bosse, R. Duell, A. Z. Memon et al., "A multi-agent model for emotion contagion spirals integrated within a supporting ambient agent model," in Principles of Practice in Multi-Agent Systems, pp. 48-67, Springer, 2009.

[6] E. Hatfield and J. T. Cacioppo, Emotional Contagion, Cambridge University Press, Cambridge, UK, 1994.

[7] S. G. Barsade, "The ripple effect: emotional contagion and its influence on group behavior," Administrative Science Quarterly, vol. 47, no. 4, pp. 644-768, 2002. 
[8] R. M. Anderson, R. M. May, and B. Anderson, Infectious Diseases of Humans: Dynamics and Control, Wiley, 1992.

[9] H. W. Hethcote and P. van den Driessche, "An SIS epidemic model with variable population size and a delay," Journal of Mathematical Biology, vol. 34, no. 2, pp. 177-194, 1995.

[10] B. Shulgin, L. Stone, and Z. Agur, "Pulse vaccination strategy in the SIR epidemic model," Bulletin of Mathematical Biology, vol. 60, no. 6, pp. 1123-1148, 1998.

[11] E. Beretta and Y. Takeuchi, "Global stability of an SIR epidemic model with time delays," Journal of Mathematical Biology, vol. 33, no. 3, pp. 250-260, 1995.

[12] P. S. Dodds and D. J. Watts, "A generalized model of social and biological contagion," Journal of Theoretical Biology, vol. 232, no. 4, pp. 587-604, 2005.

[13] A. L. Hill, D. G. Rand, M. A. Nowak, and N. A. Christakis, "Emotions as infectious diseases in a large social network: the SISa model," Proceedings of the Royal Society B: Biological Sciences, vol. 277, no. 1701, pp. 3827-3835, 2010.

[14] R. M. May and A. L. Lloyd, "Infection dynamics on scale-free networks," Physical Review E, vol. 64, no. 6, Article ID 066112, 2001.

[15] R. Pastor-Satorras and A. Vespignani, "Epidemic spreading in scale-free networks," Physical Review Letters, vol. 86, no. 14, pp. 3200-3203, 2001.

[16] V. M. Eguíluz and K. Klemm, "Epidemic threshold in structured scale-free networks," Physical Review Letters, vol. 89, no. 10, Article ID 108701, 2002.

[17] M. Boguñá and R. Pastor-Satorras, "Epidemic spreading in correlated complex networks," Physical Review E, vol. 66, no. 4, Article ID 047104, 2002.

[18] D. Centola and M. Macy, "Complex contagions and the weakness of long ties," The American Journal of Sociology, vol. 113, no. 3, pp. 702-734, 2007.

[19] P. S. Dodds and J. L. Payne, "Analysis of a threshold model of social contagion on degree-correlated networks," Physical Review E, vol. 79, no. 6, Article ID 066115, 2009.

[20] R. Pastor-Satorras and A. Vespignani, "Epidemic dynamics and endemic states in complex networks," Physical Review E, vol. 63, no. 6, Article ID 066117, 2001.

[21] L. Zhao, J. Wang, R. Huang et al., "Sentiment contagion in complex networks," Physica A: Statistical Mechanics and Its Applications, vol. 394, pp. 17-23, 2014.

[22] J. Tsai, E. Bowring, S. Marsella, and M. Tambe, "Empirical evaluation of computational emotional contagion models," in Proceedings of the 10th International Conference on Intelligent Virtual Agents (IVA '11), pp. 384-397, 2011.

[23] G. Pereira, J. Dimas, R. Prada, P. A. Santos, and A. Paiva, "A generic emotional contagion computational model," Lecture Notes in Computer Science, vol. 6974, no. 1, pp. 256-266, 2011.

[24] L. Fu, W. Song, W. Lv, and S. Lo, "Simulation of emotional contagion using modified SIR model: a cellular automaton approach," Physica A: Statistical Mechanics and Its Applications, vol. 405, pp. 380-391, 2014.

[25] F. Wen, Z. He, and X. Chen, "Investors' risk preference characteristics and conditional skewness," Mathematical Problems in Engineering, vol. 2014, Article ID 814965, 14 pages, 2014.

[26] F. Wen, Z. He, X. Gong, and A. Liu, "Investors'risk preference characteristics based on different reference point," Discrete
Dynamics in Nature and Society, vol. 2014, Article ID 158386, 9 pages, 2014.

[27] Y. Hudson and C. J. Green, "Born in the USA? Contagious investor sentiment and UK equity returns," Working Paper, 2013.

[28] Y. Lee, A. L. Tucker, D. K. Wang, and H.-T. Pao, "Global contagion of market sentiment during the US subprime crisis," Global Finance Journal, vol. 25, no. 1, pp. 17-26, 2014.

[29] Y. Bai, "Cross-border sentiment: an empirical analysis on EU stock markets," Applied Financial Economics, vol. 24, no. 4, pp. 259-290, 2014.

[30] M. Baker, J. Wurgler, and Y. Yuan, "Global, local, and contagious investor sentiment," Journal of Financial Economics, vol. 104, no. 2, pp. 272-287, 2012.

[31] C. Chang, R. Faff, and C. Y. Hwang, "Local and global sentiment effects, and the role of legal, information and trading," Working Paper, 2012.

[32] C. Huang, C. Peng, X. Chen, and F. Wen, "Dynamics analysis of a class of delayed economic model," Abstract and Applied Analysis, vol. 2013, Article ID 962738, 12 pages, 2013.

[33] C. Huang, H. Kuang, X. Chen, and F. Wen, "An LMI approach for dynamics of switched cellular neural networks with mixed delays," Abstract and Applied Analysis, vol. 2013, Article ID 870486, 8 pages, 2013.

[34] G. Qin, C. Huang, Y. Xie et al., "Asymptotic behavior for thirdorder quasi-linear differential equations," Advances in Difference Equations, vol. 2013, no. 1, article 305, 2013.

[35] N. S. Tay, "Social network characteristics and the evolution of investor sentiment," in Agent-Based Approaches in Economic and Social Complex Systems V, vol. 6 of Springer Series on Agent Based Social Systems, pp. 207-217, Springer, 2009.

[36] L. Cen, H. Lu, and L. Yang, "Investor sentiment, disagreement, and the breadth-return relationship," Management Science, vol. 59, no. 5, pp. 1076-1091, 2013.

[37] J. Liu, L. Yan, and C. Ma, "Pricing options and convertible bonds based on an actuarial approach," Mathematical Problems in Engineering, vol. 2013, Article ID 676148, 9 pages, 2013.

[38] F. Wen and X. Yang, "Skewness of return distribution and coefficient of risk premium," Journal of Systems Science \& Complexity, vol. 22, no. 3, pp. 360-371, 2009.

[39] F. Wen, X. Gong, Y. Chao, and X. Chen, "The effects of prior outcomes on risky choice: evidence from the stock market," Mathematical Problems in Engineering, vol. 2014, Article ID 272518, 8 pages, 2014. 


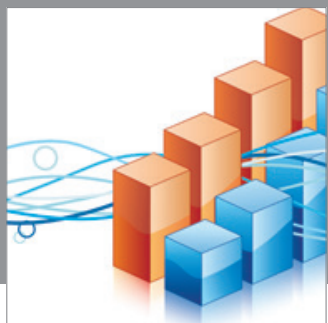

Advances in

Operations Research

mansans

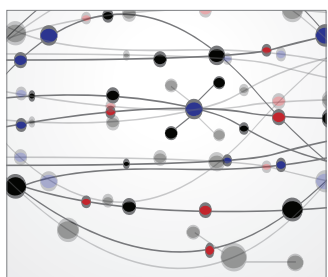

The Scientific World Journal
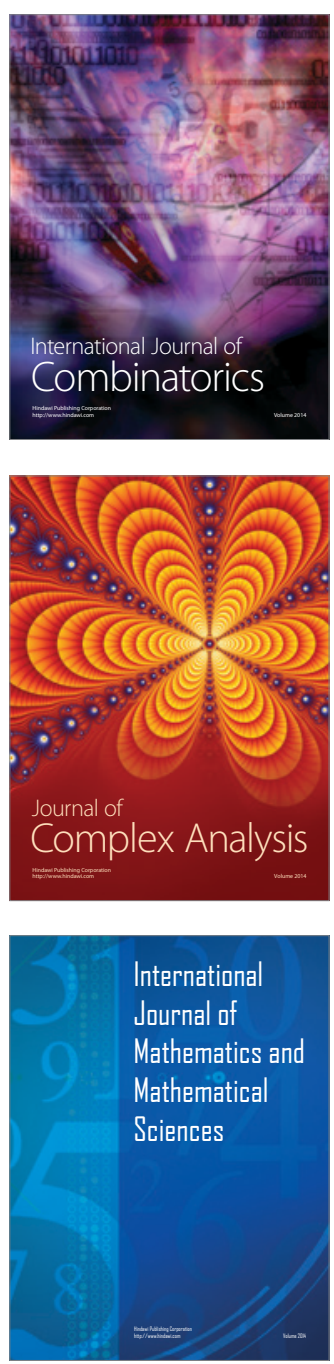
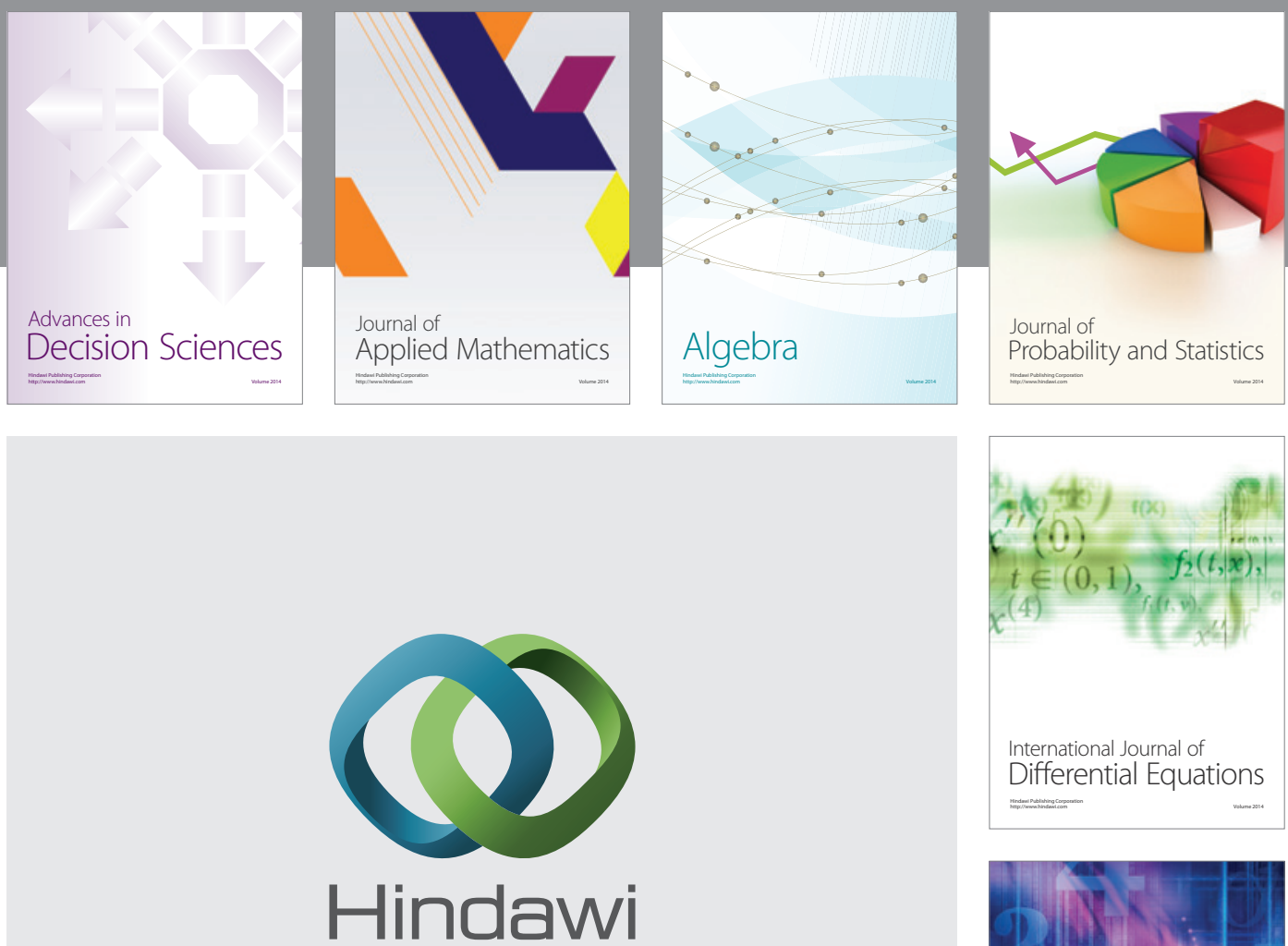

Submit your manuscripts at http://www.hindawi.com
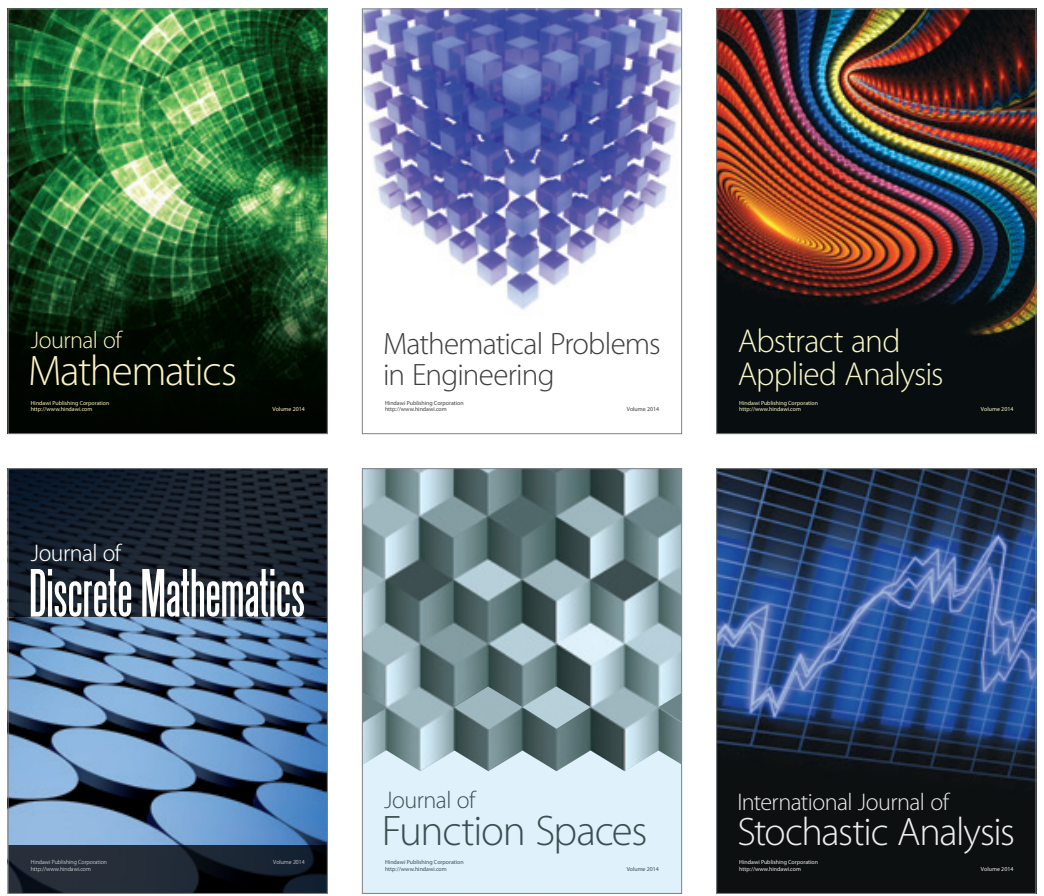

Journal of

Function Spaces

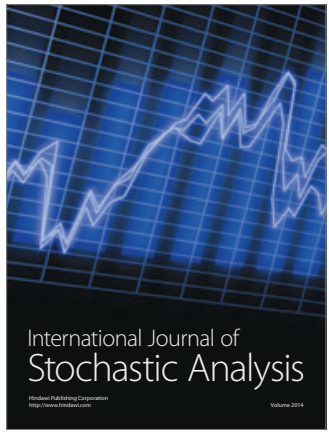

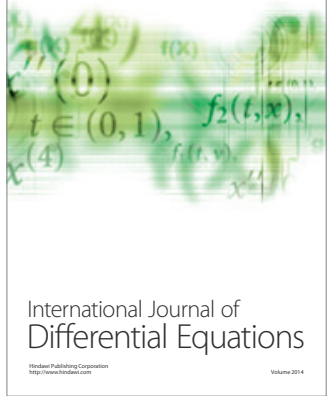
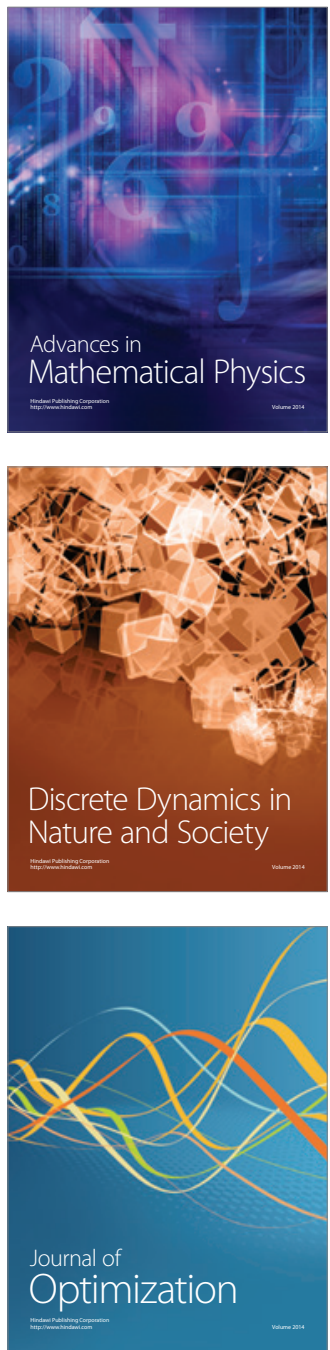